\title{
Collective Excitations of Supersymmetric Plasma
}

\author{
Alina Czajka \\ Institute of Physics, Jan Kochanowski University, 25-406 Kielce, Poland \\ Stanisław Mrówczyński \\ Institute of Physics, Jan Kochanowski University, 25-406 Kielce, Poland and \\ Sottan Institute for Nuclear Studies, 00-681 Warsaw, Poland
}

(Dated: January 24, 2011)

\begin{abstract}
Collective excitations of $\mathcal{N}=1$ supersymmetric electromagnetic plasma are studied. Since the Keldysh-Schwinger approach is used, not only equilibrium but also non-equilibrium plasma, which is assumed to be ultrarelativistic, is under consideration. The dispersion equations of photon, photino, electron, and selectron modes are written down and the self-energies, which enter the equations, are computed in the Hard Loop Approximation. The self-energies are discussed in the context of effective action which is also given. The photon modes and electron ones appear to be the same as in the usual ultrarelativistic plasma of electrons, positrons and photons. The photino modes coincide with the electron ones and the selectron modes are as of a free relativistic massive particle.
\end{abstract}

\section{INTRODUCTION}

Supersymmetry is commonly believed to be a symmetry of Nature at a sufficiently high energy scale. Obviously the symmetry must be broken, as the superpartners of particles, which constitute the Standard Model, are not seen. Experiments at the Large Hadron Collider might soon provide evidence of superparticles, but even if this is not the case, supersymmetry has proved to be a very useful concept of theoretical physics. The conjectured equivalence known as the AdS/CFT duality - of the five-dimensional gravity in the anti de Sitter geometry and the conformal field theories, see the review [1] and the lecture notes [2] as an introduction, stimulated a great interest in the $\mathcal{N}=4$ supersymmetric Yang-Mills theory. The duality has provided a unique tool to study strongly coupled field theories, as the gravitational constant is inversely proportional to the coupling constant of dual conformal field theory and thus some problems of strongly coupled field theories can be solved via weakly coupled gravity. Some intriguing results have been obtained in this way, see the reviews 3, 4, but relevance of the results for non-supersymmetric theories, which are of our actual interest, remains an open issue. One asks how properties of the supersymmetric quark-gluon plasma governed by $\mathcal{N}=4$ SUSY QCD are related to those of the usual quark-gluon plasma experimentally studied in relativistic heavy-ion collisions. While such a comparison is, in general, a difficult task, some comparative analyses have been done in the domain of weak coupling where perturbative methods are applicable [5] 9 . In particular, the paper 9 discusses the dispersion relation of quarks and squarks in equilibrium plasma using the imaginary-time formalism. It is also the aim of this paper to compare collective excitations of supersymmetric plasma to those of a non-supersymmetric counterpart. However, we study non-equilibrium plasmas where the spectrum of excitations is much richer than the equilibrium spectrum. In particular, there are unstable modes which dominate the plasma's dynamics. To simplify our analysis we consider the supersymmetric $\mathcal{N}=1$ electromagnetic plasma instead of manybody $\mathcal{N}=4$ SUSY QCD.

There is also another reason for our interest in weakly coupled supersymmetric plasma. When the plasma is homogeneous but its momentum distribution is anisotropic, there are instabilities in the gluon sector of quark-gluon plasma or in the photon sector of an electromagnetic one, see e.g. the review [10. Although a general proof is missing, there seem to be no unstable modes in the fermion sector of quarks and electrons, respectively, 11, 12. One wonders what happens in the supersymmetric plasma. Even though the supersymmetry is well known to be broken at a finite temperature or density, one can still speculate that a rudimentary symmetry induces instability in the photino sector when the photon modes are unstable. We test the hypothesis in this paper.

We start our considerations by writing down the lagrangian of supersymmetric $\mathcal{N}=1$ massless QED. In Sec. III the general dispersion equations of photons, photinos, electrons, and selectrons are written down and the self-energies, which enter the equations, are obtained in the subsequent section. The computation is performed within the KeldyshSchwinger approach which allows one to study equilibrium and non-equilibrium systems. Various Green's functions of Keldysh-Schwinger formalism are collected in the Appendix. Since we are interested in collective modes, the self-energies are found in the long wavelength limit using the Hard Loop approach which for equilibrium systems is reviewed in [13 15] while the generalization to anisotropic ones is given in [16]. The self-energies, which are computed, are also discussed in the context of Hard Loop effective action which was first derived for equilibrium plasmas in [17. 19, see also 20, 21, and generalized to anisotropic ones in [22, 23. Finally, in Sec. VI we discuss the collective modes and compare them to those of ultrarelativistic QED plasma of electrons, positrons and photons. We use the natural 
system of units with $c=\hbar=k_{B}=1$; the signature of the metric tensor is $(+---)$.

\section{II. $\mathcal{N}=1$ SUSY QED}

The lagrangian of $\mathcal{N}=1$ SUSY QED is known, see e.g. [24], to be

$$
\begin{aligned}
\mathcal{L}= & -\frac{1}{4} F^{\mu \nu} F_{\mu \nu}+i \bar{\Psi} \not D \Psi+\frac{i}{2} \bar{\Lambda} \not \partial \Lambda+\left(D_{\mu} \phi_{L}\right)^{*}\left(D^{\mu} \phi_{L}\right)+\left(D_{\mu}^{*} \phi_{R}\right)\left(D^{\mu} \phi_{R}^{*}\right) \\
& +\sqrt{2} e\left(\bar{\Psi} P_{R} \Lambda \phi_{L}-\bar{\Psi} P_{L} \Lambda \phi_{R}^{*}+\phi_{L}^{*} \bar{\Lambda} P_{L} \Psi-\phi_{R} \bar{\Lambda} P_{R} \Psi\right)-\frac{e^{2}}{2}\left(\phi_{L}^{*} \phi_{L}-\phi_{R}^{*} \phi_{R}\right)^{2},
\end{aligned}
$$

where the strength tensor $F^{\mu \nu}$ is expressed through the electromagnetic four-potential $A^{\mu}$ as $F^{\mu \nu} \equiv \partial^{\mu} A^{\nu}-\partial^{\nu} A^{\mu}$ and the covariant derivative is $D^{\mu} \equiv \partial^{\mu}+i e A^{\mu} ; \Lambda$ is the Majorana bispinor photino field, $\Psi$ is the Dirac bispinor electron field, $\phi_{L}$ and $\phi_{R}$ are the scalar left selectron and right selectron fields; the projectors $P_{L}$ and $P_{R}$ are defined in a standard way $P_{L} \equiv \frac{1}{2}\left(1-\gamma_{5}\right)$ and $P_{R} \equiv \frac{1}{2}\left(1+\gamma_{5}\right)$. Since we are interested in ultrarelativistic plasmas, the mass terms are neglected in the lagrangian. We note that the quark-gluon plasma, as studied in relativistic heavy-ion collisions, is ultrarelativistic and quark masses are usually safely ignored.

\section{DISPERSION EQUATIONS}

Dispersion equations determine dispersion relations of quasi-particle excitations. Below we write down the dispersion equation of quasi-photons, quasi-electrons, quasi-photinos, and quasi-selectrons.

\section{A. Photons}

Since the equation of motion of the electromagnetic field $A^{\mu}(k)$ is of the form

$$
\left[k^{2} g^{\mu \nu}-k^{\mu} k^{\nu}-\Pi^{\mu \nu}(k)\right] A_{\nu}(k)=0,
$$

where $\Pi^{\mu \nu}(k)$ is the retarded polarization tensor and $k \equiv(\omega, \mathbf{k})$ is the four-momentum, the general photon dispersion equation is

$$
\operatorname{det}\left[k^{2} g^{\mu \nu}-k^{\mu} k^{\nu}-\Pi^{\mu \nu}(k)\right]=0 \text {. }
$$

Equivalently, the dispersion relations are given by positions of poles of effective photon propagator. Because of the transversality of $\Pi^{\mu \nu}\left(k_{\mu} \Pi^{\mu \nu}(k)=0\right)$, which is required by gauge covariance, not all components of $\Pi^{\mu \nu}$ are independent from each other and consequently the dispersion equation (3) can be much simplified by expressing the polarization tensor through the dielectric tensor $\varepsilon^{i j}(k)$.

\section{B. Electrons}

The electron field $\psi(k)$ obeys the equation

$$
[\not k-\Sigma(k)] \psi(k)=0,
$$

where $\Sigma(k)$ is the retarded electron self-energy, and thus the dispersion equation is

$$
\operatorname{det}[\not k-\Sigma(k)]=0 \text {. }
$$

Further on we assume that the spinor structure of $\Sigma(k)$ is

$$
\Sigma(k)=\gamma^{\mu} \Sigma_{\mu}(k) .
$$

Then, substituting the expression (6) into Eq. (5) and computing the determinant as explained in Appendix 1 of [25], we get

$$
\left[\left(k^{\mu}-\Sigma^{\mu}(k)\right)\left(k_{\mu}-\Sigma_{\mu}(k)\right)\right]^{2}=0
$$




\section{Photinos}

The photino equation of motion is

$$
[\not k-\tilde{\Pi}(k)] \Lambda(k)=0
$$

where $\Lambda$ is the photino Majorana bispinor and $\tilde{\Pi}$ is the retarded self-energy. The dispersion equation is

$$
\operatorname{det}[\not k-\tilde{\Pi}(k)]=0 \text {. }
$$

Since the expected spinor structure of $\tilde{\Pi}(k)$ is analogous to that given by Eq. $(6$, the dispersion equation coincides with Eq. 7).

\section{Selectrons}

The selectron fields $\phi_{L}(k)$ and $\phi_{R}(k)$ obey the Klein-Gordon equation

$$
\left[k^{2}+\tilde{\Sigma}_{L, R}(k)\right] \phi_{L, R}(k)=0
$$

where $\tilde{\Sigma}_{L, R}(k)$ is the retarded self-energy of left or right selectrons. The dispersion equation is

$$
k^{2}+\tilde{\Sigma}_{L, R}(k)=0
$$

\section{SELF-ENERGIES}

In this section we compute the self-energies which enter the dispersion equations $(3,5,5,11)$. The plasma is assumed to be homogeneous but the momentum distribution is, in general, different from equilibrium one. Therefore, we use the the Keldysh-Schwinger formalism and the free Green's functions, which are labeled with the indices,,$+->,<$, sym, are collected in the Appendix. The computation is performed within the Hard Loop approach, see the reviews [13 15], which was generalized to anisotropic systems in [16]. The plasma is assumed to be ultrarelativistic and thus masses of electrons and selectrons are neglected. We also assume that the system is neutral and that the distribution function of electrons $\left(f_{e}(\mathbf{p})\right)$ equals the distribution function of positrons $\left(\bar{f}_{e}(\mathbf{p})\right)$. Analogous equality is assumed for selectrons: $f_{s}(\mathbf{p})=\bar{f}_{s}(\mathbf{p})$. The additional assumption is that both left and right selectrons are described by the same function $f_{s}(\mathbf{p})$.

\section{A. Polarization tensor}

The polarization tensor $\Pi^{\mu \nu}$ can be defined by means of the Dyson-Schwinger equation

$$
i \mathcal{D}^{\mu \nu}(k)=i D^{\mu \nu}(k)+i D^{\mu \rho}(k) i \Pi_{\rho \sigma}(k) i \mathcal{D}^{\sigma \nu}(k)
$$

where $\mathcal{D}^{\mu \nu}$ and $D^{\mu \nu}$ is the interacting and free photon propagator, respectively. The lowest order contributions to $\Pi^{\mu \nu}$ are given by the three diagrams shown in Fig. 1. The solid, wavy and dashed lines denote, respectively, electron, photon and selectron fields.

\section{Electron loop}

Applying the Feynman rules of the Keldysh-Schwinger formalism, which are discussed in e.g. Sec. 8 of [25], the contribution to $\Pi_{\mu \nu}^{\lessgtr}$ from the electron loop corresponding to the graph from Fig. 1 a is immediately written down in the coordinate space as

$$
i_{(a)} \Pi_{\mu \nu}^{\lessgtr}(x)=(-1)(-i e)^{2} \operatorname{Tr}\left[\gamma_{\mu} i S^{\lessgtr}(x) \gamma_{\nu} i S^{\gtrless}(-x)\right],
$$


a)

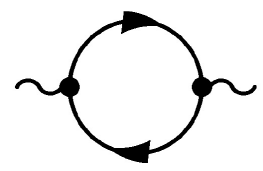

b)

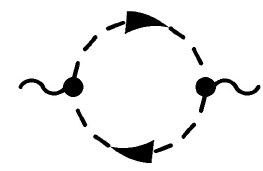

c)

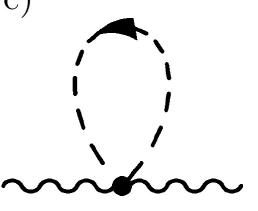

FIG. 1: Contributions to the photon self-energy.

where the factor $(-1)$ occurs due to the fermion loop. It gives

$$
{ }_{(a)} \Pi_{\mu \nu}^{\lessgtr}(x)=-i e^{2} \operatorname{Tr}\left[\gamma_{\mu} S^{\lessgtr}(x) \gamma_{\nu} S^{\gtrless}(-x)\right] .
$$

Since

$$
\Pi_{\mu \nu}^{ \pm}(x)= \pm \Theta\left( \pm x_{0}\right)\left(\Pi_{\mu \nu}^{>}(x)-\Pi_{\mu \nu}^{<}(x)\right), \quad S^{ \pm}(x)= \pm \Theta\left( \pm x_{0}\right)\left(S^{>}(x)-S^{<}(x)\right)
$$

the retarded polarization tensor ${ }_{(a)} \Pi_{\mu \nu}^{+}(x)$ is found as

$$
{ }_{(a)} \Pi_{\mu \nu}^{+}(x)=i \frac{e^{2}}{2} \operatorname{Tr}\left[\gamma_{\mu} S^{+}(x) \gamma_{\nu} S^{\mathrm{sym}}(-x)+\gamma_{\mu} S^{\mathrm{sym}}(x) \gamma_{\nu} S^{-}(-x)\right] .
$$

In the momentum space it reads

$$
{ }_{(a)} \Pi^{\mu \nu}(k)=i \frac{e^{2}}{2} \int \frac{d^{4} p}{(2 \pi)^{4}} \operatorname{Tr}\left[\gamma^{\mu} S^{+}(p+k) \gamma^{\nu} S^{\mathrm{sym}}(p)+\gamma^{\mu} S^{\mathrm{sym}}(p) \gamma^{\nu} S^{-}(p-k)\right] .
$$

The index + of the polarization tensor from Eq. 17 is dropped. Further on, we will consider only the retarded self-energies and thus the index + will not be used.

Substituting the functions $S^{ \pm}$A.9 and $S^{\text {sym }}$ A.12 into Eq. (17), one finds

$$
\begin{aligned}
{ }_{(a)} \Pi^{\mu \nu}(k)= & -\frac{e^{2}}{4} \int \frac{d^{3} p}{(2 \pi)^{3}} \frac{2 f_{e}(\mathbf{p})-1}{E_{p}} \\
& \times \operatorname{Tr}\left[\left(\frac{\gamma^{\mu}(\not p+\not k) \gamma^{\nu} \not p+\gamma^{\mu} \not p \gamma^{\nu}(\not p+\not k)}{(p+k)^{2}+i \operatorname{sgn}\left((p+k)_{0}\right) 0^{+}}+\frac{\gamma^{\mu} \not p \gamma^{\nu}(\not p-\not k)+\gamma^{\mu}(\not p-\not k) \gamma^{\nu} \not p}{(p-k)^{2}-i \operatorname{sgn}\left((p-k)_{0}\right) 0^{+}}\right)\right],
\end{aligned}
$$

where after performing the integration over $p_{0}$, the momentum $\mathbf{p}$ was changed into $-\mathbf{p}$ in the positron contribution. It was also assumed that $f_{e}(\mathbf{p})=\bar{f}_{e}(\mathbf{p})$.

Computing the traces of gamma matrices and taking into account that $p^{2}=0$, one finds

$$
\begin{aligned}
{ }_{(a)} \Pi^{\mu \nu}(k)= & -2 e^{2} \int \frac{d^{3} p}{(2 \pi)^{3}} \frac{2 f_{e}(\mathbf{p})-1}{E_{p}} \\
& \times\left(\frac{2 p^{\mu} p^{\nu}+k^{\mu} p^{\nu}+p^{\mu} k^{\nu}-g^{\mu \nu}(k \cdot p)}{(p+k)^{2}+i \operatorname{sgn}\left((p+k)_{0}\right) 0^{+}}+\frac{2 p^{\mu} p^{\nu}-k^{\mu} p^{\nu}-p^{\mu} k^{\nu}+g^{\mu \nu}(k \cdot p)}{(p-k)^{2}-i \operatorname{sgn}\left((p-k)_{0}\right) 0^{+}}\right) .
\end{aligned}
$$

We are interested in collective modes which occur when the wavelength of a quasi-particle is much bigger than a characteristic interparticle distance in the plasma. Thus, we look for the polarization tensor at $k^{\mu} \ll p^{\mu}$ which is the condition of the Hard Loop Approximation for anisotropic systems [16, 23. The approximation is implemented by observing that

$$
\begin{gathered}
\frac{1}{(p+k)^{2}+i 0^{+}}+\frac{1}{(p-k)^{2}-i 0^{+}}=\frac{2 k^{2}}{\left(k^{2}\right)^{2}-4(k \cdot p)^{2}-i \operatorname{sgn}(k \cdot p) 0^{+}} \approx-\frac{1}{2} \frac{k^{2}}{\left(k \cdot p+i 0^{+}\right)^{2}} \\
\frac{1}{(p+k)^{2}+i 0^{+}}-\frac{1}{(p-k)^{2}-i 0^{+}}=\frac{4(k \cdot p)}{\left(k^{2}\right)^{2}-4(k \cdot p)^{2}-i \operatorname{sgn}(k \cdot p) 0^{+}} \approx \frac{k \cdot p}{\left(k \cdot p+i 0^{+}\right)^{2}} .
\end{gathered}
$$


We note that $(p+k)_{0}>0,(p-k)_{0}>0$ for $p^{\mu} \gg k^{\mu}$. With the above formulas Eq. 19 gives

$$
{ }_{(a)} \Pi^{\mu \nu}(k)=2 e^{2} \int \frac{d^{3} p}{(2 \pi)^{3}} \frac{2 f_{e}(\mathbf{p})-1}{E_{p}} \frac{k^{2} p^{\mu} p^{\nu}-\left(k^{\mu} p^{\nu}+p^{\mu} k^{\nu}-g^{\mu \nu}(k \cdot p)\right)(k \cdot p)}{\left(k \cdot p+i 0^{+}\right)^{2}},
$$

which is the well-known form of polarization tensor of photons and of gluons in ultrarelativistic plasmas, see e.g. the reviews [10, 14]. As seen, ${ }_{(a)} \Pi^{\mu \nu}(k)$ is symmetric $\left({ }_{(a)} \Pi^{\mu \nu}(k)={ }_{(a)} \Pi^{\nu \mu}(k)\right)$ and transverse $\left(k_{\mu(a)} \Pi^{\mu \nu}(k)=0\right)$ as required by the gauge invariance.

\section{Selectron loop}

The contribution to the polarization tensor coming from the selectron loop depicted in Fig. 1p is given by an appropriately modified Eq. (17) that is

$$
{ }_{(b)} \Pi^{\mu \nu}(k)=-i \frac{e^{2}}{2} \int \frac{d^{4} p}{(2 \pi)^{4}}\left[(2 p+k)^{\mu}(2 p+k)^{\nu} \tilde{S}^{+}(p+k) \tilde{S}^{\mathrm{sym}}(p)+(2 p-k)^{\mu}(2 p-k)^{\nu} \tilde{S}^{\mathrm{sym}}(p) \tilde{S}^{-}(p-k)\right] .
$$

The sign is different than in Eq. (17), as we deal here with the boson not the fermion loop. Substituting the functions $\tilde{S}^{ \pm}$and $\tilde{S}^{\text {sym }}$ given by Eqs. A.19. A.22 into Eq. 21, one finds

$$
{ }_{(b)} \Pi^{\mu \nu}(k)=-\frac{e^{2}}{2} \int \frac{d^{3} p}{(2 \pi)^{3}} \frac{2 f_{s}(\mathbf{p})+1}{E_{p}}\left[\frac{(2 p+k)^{\mu}(2 p+k)^{\nu}}{(p+k)^{2}+i \operatorname{sgn}\left((p+k)_{0}\right) 0^{+}}+\frac{(2 p-k)^{\mu}(2 p-k)^{\nu}}{(p-k)^{2}-i \operatorname{sgn}\left((p-k)_{0}\right) 0^{+}}\right],
$$

where the change $\mathbf{p} \rightarrow-\mathbf{p}$ was made in the antiselectron part and we assumed that $\bar{f}_{s}(\mathbf{p})=f_{s}(\mathbf{p})$. After adopting the Hard Loop Approximation Eq. 222) gives

$$
{ }_{(b)} \Pi^{\mu \nu}(k)=e^{2} \int \frac{d^{3} p}{(2 \pi)^{3}} \frac{2 f_{s}(\mathbf{p})+1}{E_{p}} \frac{k^{2} p^{\mu} p^{\nu}-\left(p^{\mu} k^{\nu}+k^{\mu} p^{\nu}\right)(k \cdot p)}{\left(k \cdot p+i 0^{+}\right)^{2}} .
$$

\section{Selectron tadpole}

The contribution to the polarization tensor coming from the selectron tadpole depicted in Fig. 11 is

$$
i_{(c)} \Pi^{\mu \nu}(k)=2 i e^{2} g^{\mu \nu} \int \frac{d^{4} p}{(2 \pi)^{4}} i \tilde{S}^{<}(p) .
$$

Substituting the function $\tilde{S}^{<}$given by Eq. A.21 into Eq. 24, one finds

$$
{ }_{(c)} \Pi^{\mu \nu}(k)=e^{2} g^{\mu \nu} \int \frac{d^{3} p}{(2 \pi)^{3}} \frac{2 f_{s}(\mathbf{p})+1}{E_{p}},
$$

where the equality $\bar{f}_{s}(\mathbf{p})=f_{s}(\mathbf{p})$ was assumed.

We get the complete contribution from a single selectron field to the polarization tensor by summing the contributions from the selectron loop and the selectron tadpole. Thus, one finds

$$
{ }_{(b+c)} \Pi^{\mu \nu}(k)=e^{2} \int \frac{d^{3} p}{(2 \pi)^{3}} \frac{2 f_{s}(\mathbf{p})+1}{E_{p}} \frac{k^{2} p^{\mu} p^{\nu}-\left(p^{\mu} k^{\nu}+k^{\mu} p^{\nu}-g^{\mu \nu}(k \cdot p)\right)(k \cdot p)}{\left(k \cdot p+i 0^{+}\right)^{2}} .
$$

As seen, it is of exactly the same form as the electron contribution given by Eq. 20 - it is symmetric and transversal. Actually, the expression 20 is the polarization tensor of scalar QED, which for equilibrium plasma was discussed in e.g. [26] using the imaginary-time formalism. Since there are two selectron fields in $\mathcal{N}=1$ SUSY QED, the expression 26) should be multiplied by a factor of 2 to get the complete selectron contribution to the polarization tensor. 
a)

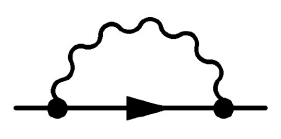

b)

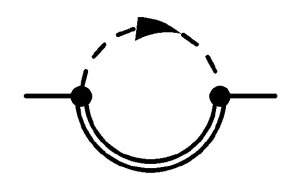

FIG. 2: Contributions to the electron self-energy.

\section{Final result}

Combining the electron (20) and selectron 26) contributions, we get the final expression of the polarization tensor

$$
\Pi^{\mu \nu}(k)=4 e^{2} \int \frac{d^{3} p}{(2 \pi)^{3}} \frac{f_{e}(\mathbf{p})+f_{s}(\mathbf{p})}{E_{p}} \frac{k^{2} p^{\mu} p^{\nu}-\left(p^{\mu} k^{\nu}+k^{\mu} p^{\nu}-g^{\mu \nu}(k \cdot p)\right)(k \cdot p)}{\left(k \cdot p+i 0^{+}\right)^{2}} .
$$

As seen, $\Pi^{\mu \nu}(k)$ vanishes in the vacuum limit when $f_{e}, f_{s} \rightarrow 0$. This is a nice feature of supersymmetric plasma. In the non-supersymmetric counterpart, the polarization tensor is given by Eq. (20) where the vacuum contribution diverges and it requires a special treatment. Up to the vacuum contribution, the polarization tensor of supersymmetric plasma and of its non-supersymmetric counterpart has the same structure.

\section{B. Electron self-energy}

The electron self-energy $\Sigma$ can be defined by means of the Dyson-Schwinger equation

$$
i \mathcal{S}(k)=i S(k)+i S(k)(-i \Sigma(k)) i \mathcal{S}(k),
$$

where $\mathcal{S}$ and $S$ is the interacting and free propagator, respectively. The lowest order contributions to $\Sigma$ are given by two diagrams shown in Fig. 2. The solid, wavy, dashed and double-solid lines denote, respectively, electron, photon, selectron and photino fields.

\section{Electron-photon loop}

The contribution to the electron self-energy corresponding to the graph depicted in Fig. $2 \mathrm{a}$ is given by an appropriately modified Eq. (17) that is

$$
-i_{(a)} \Sigma(k)=(-i e)^{2} \frac{1}{2} \int \frac{d^{4} p}{(2 \pi)^{4}}\left[\gamma^{\mu} i S^{+}(p+k) \gamma^{\nu} i D_{\mu \nu}^{\mathrm{sym}}(p)+\gamma^{\mu} i S^{\mathrm{sym}}(p) \gamma^{\nu} i D_{\mu \nu}^{-}(p-k)\right],
$$

which trivially gives

$$
{ }_{(a)} \Sigma(k)=i e^{2} \frac{1}{2} \int \frac{d^{4} p}{(2 \pi)^{4}}\left[\gamma^{\mu} S^{+}(p+k) \gamma^{\nu} D_{\mu \nu}^{\mathrm{sym}}(p)+\gamma^{\mu} S^{\mathrm{sym}}(p) \gamma^{\nu} D_{\mu \nu}^{-}(p-k)\right] .
$$

Substituting the functions $D_{\mu \nu}^{ \pm}, D_{\mu \nu}^{\mathrm{sym}}$ and $S^{ \pm}, S^{\mathrm{sym}}$ given by Eqs. A.1, A.4, A.9, A.12 into Eq. 30, one finds

$$
\begin{aligned}
\left(a_{a} \Sigma(k)=\frac{e^{2}}{2} \int \frac{d^{3} p}{(2 \pi)^{3} E_{p}}\{\right. & {\left[\frac{\not p+\not k}{(p+k)^{2}+i \operatorname{sgn}\left((p+k)_{0}\right) 0^{+}}-\frac{\not p-\not k}{(p-k)^{2}-i \operatorname{sgn}\left((p-k)_{0}\right) 0^{+}}\right]\left[2 f_{\gamma}(\mathbf{p})+1\right] } \\
& \left.-\left[\frac{\not p}{(p-k)^{2}-i \operatorname{sgn}\left((p-k)_{0}\right) 0^{+}}-\frac{\not p}{(p+k)^{2}+i \operatorname{sgn}\left((p+k)_{0}\right) 0^{+}}\right]\left[2 f_{e}(\mathbf{p})-1\right]\right\},
\end{aligned}
$$

where the change $\mathbf{p} \rightarrow-\mathbf{p}$ was made in the negative energy terms. It was also assumed that $f_{e}(\mathbf{p})=\bar{f}_{e}(\mathbf{p})$. Applying the Hard Loop Approximation, one obtains

$$
{ }_{(a)} \Sigma(k)=e^{2} \int \frac{d^{3} p}{(2 \pi)^{3}} \frac{f_{\gamma}(\mathbf{p})+f_{e}(\mathbf{p})}{E_{p}} \frac{\not p}{k \cdot p+i 0^{+}},
$$

which is the well-known form of self-energy of electrons and of quarks in ultrarelativistic plasmas, see e.g. the review [14. 


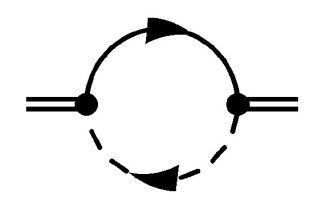

FIG. 3: Contribution to the photino self-energy.

\section{Selectron-photino loop}

Since there are two selectron fields in $\mathcal{N}=1$ SUSY QED there are two contributions to the electron self-energy corresponding to the graph depicted in Fig. $2 \mathrm{~b}$. The first one corresponding the left selectron field equals

$$
-i_{(b L)} \Sigma(k)=(-i e \sqrt{2})^{2} \frac{1}{2} \int \frac{d^{4} p}{(2 \pi)^{4}}\left[i \tilde{S}^{+}(p+k) P_{L} i \tilde{D}^{\mathrm{sym}}(p) P_{R}+i \tilde{S}^{\mathrm{sym}}(p) P_{L} i \tilde{D}^{-}(p-k) P_{R}\right],
$$

which is

$$
{ }_{(b L)} \Sigma(k)=i e^{2} \int \frac{d^{4} p}{(2 \pi)^{4}}\left[\tilde{S}^{+}(p+k) P_{L} \tilde{D}^{\mathrm{sym}}(p) P_{R}+\tilde{S}^{\mathrm{sym}}(p) P_{L} \tilde{D}^{-}(p-k) P_{R}\right] .
$$

Substituting the functions $\tilde{D}^{ \pm}, \tilde{D}^{\text {sym }}$ and $\tilde{S}^{ \pm}, \tilde{S}^{\text {sym }}$ given by Eqs. A.14, A.17, A.19, A.22 into Eq. 34, one finds in the Hard Loop Approximation the following result

$$
{ }_{(b L)} \Sigma(k)=e^{2} \int \frac{d^{3} p}{(2 \pi)^{3}} \frac{f_{\tilde{\gamma}}(\mathbf{p})+f_{s}(\mathbf{p})}{E_{p}} \frac{P_{L} \not p P_{R}}{k \cdot p+i 0^{+}},
$$

where we assumed that $f_{s}(\mathbf{p})=\bar{f}_{s}(\mathbf{p})$.

Computing the contribution corresponding to the graph depicted in Fig. $2 \mathrm{~b}$ with the right selectron field, we get

$$
{ }_{(b R)} \Sigma(k)=e^{2} \int \frac{d^{3} p}{(2 \pi)^{3}} \frac{f_{\tilde{\gamma}}(\mathbf{p})+f_{s}(\mathbf{p})}{E_{p}} \frac{P_{R} p P_{L}}{k \cdot p+i 0^{+}} .
$$

Because $P_{L} \not p P_{R}+P_{R} \not p P_{L}=\not p$, the total contribution given by the graph from Fig. 2 b equals

$$
{ }_{(b)} \Sigma(k)=e^{2} \int \frac{d^{3} p}{(2 \pi)^{3}} \frac{f_{\tilde{\gamma}}(\mathbf{p})+f_{s}(\mathbf{p})}{E_{p}} \frac{\not p}{k \cdot p+i 0^{+}} .
$$

\section{Final result}

The sum of expressions 32 and (37) gives the complete electron self-energy

$$
\Sigma(k)=e^{2} \int \frac{d^{3} p}{(2 \pi)^{3}} \frac{f_{\gamma}(\mathbf{p})+f_{e}(\mathbf{p})+f_{\tilde{\gamma}}(\mathbf{p})+f_{s}(\mathbf{p})}{E_{p}} \frac{\not p}{k \cdot p+i 0^{+}} .
$$

As seen, the electron self-energy has the same structure for the supersymmetric plasma and for its non-supersymmetric counterpart.

\section{Photino self-energy}

The photino self-energy Ĩ can be defined by means of the Dyson-Schwinger equation

$$
i \tilde{\mathcal{D}}(k)=i \tilde{D}(k)+i \tilde{D}(k)(-i \tilde{\Pi}(k)) i \tilde{\mathcal{D}}(k)
$$

where $\tilde{\mathcal{D}}$ and $\tilde{D}$ is the interacting and free photino propagator, respectively. The lowest order contribution to $\tilde{\Pi}$ is given by the diagram shown in Fig. 3. The solid, dashed and double-solid lines denote, respectively, electron, selectron 
and photino fields. Since there are two selectron fields in $\mathcal{N}=1$ SUSY QED there are two contributions represented by the diagram corresponding to the left and right selectrons. Appropriately modifying Eq. (17), one gets

$$
-i_{(L)} \tilde{\Pi}(k)=\frac{1}{2}(-i e \sqrt{2})^{2} \int \frac{d^{4} p}{(2 \pi)^{4}}\left[P_{R} i S^{+}(p+k) P_{L} i \tilde{S}^{\mathrm{sym}}(p)+P_{R} i S^{\mathrm{sym}}(p) P_{L} i \tilde{S}^{-}(p-k)\right]
$$

where the contribution from left selectrons is taken into account. Eq. 40 is trivially manipulated to

$$
{ }_{(L)} \tilde{\Pi}(k)=i e^{2} \int \frac{d^{4} p}{(2 \pi)^{4}}\left[P_{R} S^{+}(p+k) P_{L} \tilde{S}^{\mathrm{sym}}(p)+P_{R} S^{\mathrm{sym}}(p) P_{L} \tilde{S}^{-}(p-k)\right] .
$$

Now one substitutes the functions $S^{ \pm}, S^{\text {sym }}$ and $\tilde{S}^{ \pm}, \tilde{S}^{\text {sym }}$ given by Eqs. A.9, A.12, A.19, A.22, into Eq. (41). Performing the integration over $p_{0}$ and changing $\mathbf{p}$ into $-\mathbf{p}$ in the terms representing antiparticles, we obtain

$$
\begin{aligned}
(L) \tilde{\Pi}(k)= & \frac{1}{2} e^{2} \int \frac{d^{3} p}{(2 \pi)^{3} E_{p}} \\
& \times\left\{\left[\frac{P_{R}(\not p+\not k) P_{L}}{(p+k)^{2}+i \operatorname{sgn}\left((p+k)_{0}\right) 0^{+}}-\frac{P_{R}(\not p-\not k) P_{L}}{(p-k)^{2}-i \operatorname{sgn}\left((p-k)_{0}\right) 0^{+}}\right]\left(2 f_{s}(\mathbf{p})+1\right)\right. \\
& \left.+\left[\frac{P_{R} \not p P_{L}}{(p+k)^{2}+i \operatorname{sgn}\left((p+k)_{0}\right) 0^{+}}-\frac{P_{R} \not p P_{L}}{(p-k)^{2}-i \operatorname{sgn}\left((p-k)_{0}\right) 0^{+}}\right]\left(2 f_{e}(\mathbf{p})-1\right)\right\},
\end{aligned}
$$

where we assumed that $f_{s}(\mathbf{p})=\bar{f}_{s}(\mathbf{p})$ and $f_{e}(\mathbf{p})=\bar{f}_{e}(\mathbf{p})$. Adopting the Hard Loop Approximation, one gets

$$
{ }_{(L)} \tilde{\Pi}(k)=e^{2} \int \frac{d^{3} p}{(2 \pi)^{3}} \frac{f_{s}(\mathbf{p})+f_{e}(\mathbf{p})}{E_{p}} \frac{P_{R} \not p P_{L}}{k \cdot p+i 0^{+}} .
$$

Since the contribution to the photino self-energy coming from right selectrons, which is obtained in the same way, reads

$$
{ }_{(R)} \tilde{\Pi}(k)=e^{2} \int \frac{d^{3} p}{(2 \pi)^{3}} \frac{f_{s}(\mathbf{p})+f_{e}(\mathbf{p})}{E_{p}} \frac{P_{L} \not p P_{R}}{k \cdot p+i 0^{+}},
$$

one finds, using the well-known identity $P_{R} \not p P_{L}+P_{L} \not p P_{R}=\not p$, the complete photino self-energy as

$$
\tilde{\Pi}(k)=e^{2} \int \frac{d^{3} p}{(2 \pi)^{3}} \frac{f_{s}(\mathbf{p})+f_{e}(\mathbf{p})}{E_{p}} \frac{\not p}{k \cdot p+i 0^{+}} .
$$

As seen, the photino self-energy (45) has the same structure as the electron self-energy (38.

\section{Selectron self-energy}

The selectron self-energy $\tilde{\Sigma}$ can be defined by means of the Dyson-Schwinger equation

$$
i \tilde{\mathcal{S}}(k)=i \tilde{S}(k)+i \tilde{S}(k) i \tilde{\Sigma}(k) i \tilde{\mathcal{S}}(k)
$$

where $\tilde{\mathcal{S}}$ and $\tilde{S}$ is the interacting and free propagator, respectively. The lowest order contributions to $\tilde{\Sigma}$ are given by four diagrams shown in Fig. 4. The solid, wavy, dashed and double-solid lines denote, respectively, electron, photon, selectron and photino fields. Below we compute the self-energy of left selectron. The result for right selectron is the same.

\section{Selectron tadpole}

There are two contributions represented by the graph depicted in Fig. 4 a, as the tadpole line corresponds to either left or right selectron. In the first case we have

$$
i_{(a L)} \tilde{\Sigma}_{L}(k)=-2 i e^{2} \int \frac{d^{4} p}{(2 \pi)^{4}} i \tilde{S}^{<}(p) .
$$


a)

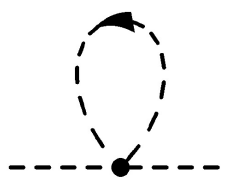

b)

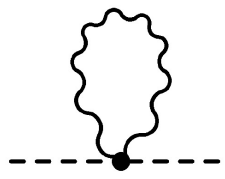

c)

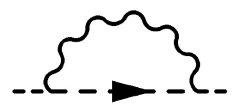

d)

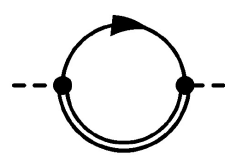

FIG. 4: Contributions to the selectron self-energy.

Substituting the function $\tilde{S}^{<}$given by Eq. A.21 into Eq. 477, one finds

$$
{ }_{(a L)} \tilde{\Sigma}_{L}(k)=-e^{2} \int \frac{d^{3} p}{(2 \pi)^{3}} \frac{2 f_{s}(\mathbf{p})+1}{E_{p}},
$$

where the equality $\bar{f}_{s}(\mathbf{p})=f_{s}(\mathbf{p})$ was assumed. The second contribution corresponding to the right-selectron field equals

$$
i_{(a R)} \tilde{\Sigma}_{L}(k)=i e^{2} \int \frac{d^{4} p}{(2 \pi)^{4}} i \tilde{S}^{<}(p),
$$

and it gives

$$
{ }_{(a R)} \tilde{\Sigma}_{L}(k)=\frac{1}{2} e^{2} \int \frac{d^{3} p}{(2 \pi)^{3}} \frac{2 f_{s}(\mathbf{p})+1}{E_{p}} .
$$

Summing up the contributions 48,49 , one finds the following complete result of the selectron tadpole

$$
{ }_{(a)} \tilde{\Sigma}_{L}(k)=-\frac{1}{2} e^{2} \int \frac{d^{3} p}{(2 \pi)^{3}} \frac{2 f_{s}(\mathbf{p})+1}{E_{p}} .
$$

\section{Photon tadpole}

The contribution to the selectron self-energy coming from the photon tadpole shown in Fig. 4b equals

$$
i_{(b)} \tilde{\Sigma}_{L}(k)=i e^{2} g^{\mu \nu} \int \frac{d^{4} p}{(2 \pi)^{4}} i D_{\mu \nu}^{<}(p),
$$

where the symmetry factor $1 / 2$ is included. Eq. (52) gives

$$
{ }_{(b)} \tilde{\Sigma}_{L}(k)=-2 e^{2} \int \frac{d^{3} p}{(2 \pi)^{3}} \frac{2 f_{\gamma}(\mathbf{p})+1}{E_{p}},
$$

when the function $D_{\mu \nu}^{<}$A.3. is substituted into Eq. 52 .

\section{Selectron-photon loop}

The contribution represented by the graph depicted in Fig. 4. equals

$$
i_{(c)} \tilde{\Sigma}_{L}(k)=\frac{1}{2}(-i e)^{2} \int \frac{d^{4} p}{(2 \pi)^{4}}\left[(p+2 k)^{\mu} i \tilde{S}_{\mu \nu}^{+}(p+k)(p+2 k)^{\nu} i D^{\mathrm{sym}}(p)+(p+k)^{\mu} i \tilde{S}_{\mu \nu}^{\mathrm{sym}}(p)(p+k)^{\nu} i D^{-}(p-k)\right],
$$

which after the substitution of the functions $D_{\mu \nu}^{ \pm}, D_{\mu \nu}^{\mathrm{sym}}$ and $\tilde{S}^{ \pm}, \tilde{S}^{\mathrm{sym}}$ in the form A.1. A.4, A.19, A.22 leads to

$$
\begin{aligned}
(c) \tilde{\Sigma}_{L}(k)= & \frac{1}{4} e^{2} \int \frac{d^{3} p}{(2 \pi)^{3} E_{p}} \\
& \times\left[\left(\frac{(p+2 k)^{2}}{(p+k)^{2}+i \operatorname{sgn}\left((p+k)_{0}\right) 0^{+}}+\frac{(p-2 k)^{2}}{(p-k)^{2}-i \operatorname{sgn}\left((p-k)_{0}\right) 0^{+}}\right)\left(2 f_{s}(\mathbf{p})+1\right)\right. \\
& \left.+\left(\frac{(p+k)^{2}}{(p-k)^{2}-i \operatorname{sgn}\left((p-k)_{0}\right) 0^{+}}+\frac{(p-k)^{2}}{(p+k)^{2}+i \operatorname{sgn}\left((p+k)_{0}\right) 0^{+}}\right)\left(2 f_{\gamma}(\mathbf{p})+1\right)\right],
\end{aligned}
$$


where we assumed that $\bar{f}_{s}(\mathbf{p})=f_{s}(\mathbf{p})$. Within the Hard Loop Approximation, one obtains

$$
{ }_{(c)} \tilde{\Sigma}_{L}(k)=\frac{e^{2}}{2} \int \frac{d^{3} p}{(2 \pi)^{3}} \frac{4 f_{\gamma}(\mathbf{p})-2 f_{s}(\mathbf{p})+1}{E_{p}} .
$$

We note that the sum of the contributions (53, 56), which equals

$$
{ }_{(b+c)} \tilde{\Sigma}_{L}(k)=-\frac{e^{2}}{2} \int \frac{d^{3} p}{(2 \pi)^{3}} \frac{4 f_{\gamma}(\mathbf{p})+2 f_{s}(\mathbf{p})+3}{E_{p}},
$$

represents the scalar self-energy of scalar QED which for equilibrium plasma was discussed in e.g. [26] within the imaginary-time formalism.

\section{Electron-photino loop}

The graph depicted in Fig. 4 $\mathrm{d}$ provides

$$
i_{(d)} \tilde{\Sigma}_{L}(k)=(-1)(-i e \sqrt{2})^{2} \frac{1}{2} \int \frac{d^{4} p}{(2 \pi)^{4}} \operatorname{Tr}\left[P_{R} i S^{+}(p+k) P_{L} i \tilde{D}^{\mathrm{sym}}(p)+P_{R} i S^{\mathrm{sym}}(p) P_{L} i \tilde{D}^{-}(p-k)\right] .
$$

Substituting the functions $\tilde{D}^{ \pm}, \tilde{D}^{\text {sym }}$ and $S^{ \pm}, S^{\text {sym }}$ given by Eqs. A.9, A.12, A.19, A.22 into Eq. (58) and repeating the same steps which were made in the previous subsections, we find in the Hard Loop Approximation the following expression

$$
(d) \tilde{\Sigma}_{L}(k)=-2 e^{2} \int \frac{d^{3} p}{(2 \pi)^{3}} \frac{f_{\tilde{\gamma}}(\mathbf{p})+f_{e}(\mathbf{p})-1}{E_{p}},
$$

where we assumed that $f_{e}(\mathbf{p})=\bar{f}_{e}(\mathbf{p})$.

\section{Final result}

The sum of contributions $51,53,56)$ and (59) gives the complete self-energy of left selectron

$$
\tilde{\Sigma}(k)=-2 e^{2} \int \frac{d^{3} p}{(2 \pi)^{3}} \frac{f_{e}(\mathbf{p})+f_{\gamma}(\mathbf{p})+f_{s}(\mathbf{p})+f_{\tilde{\gamma}}(\mathbf{p})}{E_{p}},
$$

which equals the complete self-energy of right selectron. For this reason the index $L$ is dropped. As seen, the self-energy (60) is independent of $k$ and because of supersymmetry it vanishes in the vacuum limit when all the distribution functions are zero. This is also effect of the supersymmetry that the distribution functions of electrons

and of selectrons enter the formula 60 with the coefficients equal to each other. The same is true for the distribution functions of photons and of photinos.

\section{EFFECTIVE ACTION}

The Hard Loop approach can be formulated in an elegant and compact way by using the effective action which was first derived for equilibrium plasmas in [17-19] within the thermal field theory. It was also rederived in terms of quasiclassical kinetic theory [20, 21]. Later on a generalization of the action to anisotropic systems was given in [22, 23 .

The form of self-energy constrains the possible structure of the respective effective action. Since the self-energy of a given field is the second functional derivative of the action with respect to the field, one writes

$$
\begin{aligned}
& \mathcal{L}_{2}^{(A)}(x)=\frac{1}{2} \int d^{4} y A_{\mu}(x) \Pi^{\mu \nu}(x-y) A_{\nu}(y), \\
& \mathcal{L}_{2}^{(\Psi)}(x)=\int d^{4} y \bar{\Psi}(x) \Sigma(x-y) \Psi(y)
\end{aligned}
$$




$$
\begin{aligned}
\mathcal{L}_{2}^{(\Lambda)}(x) & =\frac{1}{2} \int d^{4} y \bar{\Lambda}(x) \tilde{\Pi}(x-y) \Lambda(y), \\
\mathcal{L}_{2}^{\left(\phi_{L, R}\right)}(x) & =\int d^{4} y \phi_{L, R}^{*}(x) \tilde{\Sigma}(x-y) \phi_{L, R}(y),
\end{aligned}
$$

where the self-energies are given by the formulas $27,38,45,60$, respectively. The subscript ' 2 ' indicates that the effective actions above only generate two-point functions. To generate $n$-point functions these actions need to be extended to a gauge invariant form. In the Abelian gauge theory studied here, the actions (61, 63 64) are gauge invariant and complete. The action (62) requires a simple modification - the ordinary derivative is replaced by the covariant one in the final expression. Repeating the calculations described in detail in [23], one finds the Hard Loop effective action of $\mathcal{N}=1$ SUSY QED as

$$
\begin{aligned}
\mathcal{L}_{\mathrm{HL}}= & -\frac{1}{4} F^{\mu \nu} F_{\mu \nu}+i \bar{\Psi} \not D \Psi+\frac{i}{2} \bar{\Lambda} \not \partial \Lambda+\left(D_{\mu} \phi_{L}\right)^{*}\left(D^{\mu} \phi_{L}\right)+\left(D_{\mu}^{*} \phi_{R}\right)\left(D^{\mu} \phi_{R}^{*}\right) \\
& +\mathcal{L}_{\mathrm{HL}}^{(A)}+\mathcal{L}_{\mathrm{HL}}^{(\Psi)}+\mathcal{L}_{\mathrm{HL}}^{(\Lambda)}+\mathcal{L}_{\mathrm{HL}}^{\left(\phi_{L}\right)}+\mathcal{L}_{\mathrm{HL}}^{\left(\phi_{R}\right)},
\end{aligned}
$$

where

$$
\begin{aligned}
\mathcal{L}_{\mathrm{HL}}^{(A)} & =4 e^{2} \int \frac{d^{3} p}{(2 \pi)^{3}} \frac{f_{e}(\mathbf{p})+f_{s}(\mathbf{p})}{E_{p}} F_{\mu \nu}(x) \frac{p^{\nu} p^{\rho}}{(p \cdot \partial)^{2}} F_{\rho}{ }^{\mu}(x), \\
\mathcal{L}_{\mathrm{HL}}^{(\Psi)} & =i e^{2} \int \frac{d^{3} p}{(2 \pi)^{3}} \frac{f_{\gamma}(\mathbf{p})+f_{e}(\mathbf{p})+f_{\tilde{\gamma}}(\mathbf{p})+f_{s}(\mathbf{p})}{E_{p}} \bar{\Psi}(x) \frac{p \cdot \gamma}{p \cdot D} \Psi(x), \\
\mathcal{L}_{\mathrm{HL}}^{(\Lambda)} & =i e^{2} \int \frac{d^{3} p}{(2 \pi)^{3}} \frac{f_{s}(\mathbf{p})+f_{e}(\mathbf{p})}{E_{p}} \bar{\Lambda}(x) \frac{p \cdot \gamma}{p \cdot \partial} \Lambda(y), \\
\mathcal{L}_{\mathrm{HL}}^{\left(\phi_{L, R}\right)} & =-2 e^{2} \int \frac{d^{3} p}{(2 \pi)^{3}} \frac{f_{e}(\mathbf{p})+f_{\gamma}(\mathbf{p})+f_{s}(\mathbf{p})+f_{\tilde{\gamma}}(\mathbf{p})}{E_{p}} \phi_{L, R}^{*}(x) \phi_{L, R}(x) .
\end{aligned}
$$

The actions $61,62,63,64)$ are obtained from the self-energies but the reasoning can be turned around. As argued in [18, 19, the actions of gauge bosons (66), charged fermions (67) and charged scalars (69) are of unique gauge invariant form. Therefore, the respective self-energies can be, in principle, inferred from the known QED self-energies with some help from supersymmetry arguments. In the case of photino self-energy, which is of our main interest, the explicit computation seems to be unavoidable.

\section{COLLECTIVE MODES AND CONCLUSION}

When the self-energies computed in Sec. IV are substituted into the dispersion equations presented in Sec. III, collective modes can be found as solutions of the equations. Below we briefly discuss the photon, electron, photino and selectron excitations.

- The structure of polarization tensor 26 is such as that in the usual non-supersymmetric QED plasma. It also coincides with the gluon polarization tensor of QCD plasma. Therefore, the spectrum of collective excitations of gauge bosons is in all three cases the same. In equilibrium plasma we have the longitudinal (plasmon) mode and the transverse one which are discussed in e.g. the textbook 27. When the plasma is out of equilibrium there is a whole variety of possible collective excitations. In particular, there are unstable modes, see $e . g$. the review [10, which exponentially grow in time and strongly influence the system's dynamics.

- The form of electron self-energy (38) happens to be the same as in the usual non-supersymmetric QED plasma. The quark self-energy in QCD plasma has the same form. Therefore, we have identical spectrum of excitations of charged fermions in the three systems. In equilibrium plasma there two modes, see in e.g. the textbook [27, of opposite helicity over chirality ratio. One mode corresponds to the positive energy fermion, another one, sometimes called a plasmino, is a specific medium effect. In non-equilibrium plasma the spectrum of fermion collective excitations changes but no unstable modes have been found even for an extremely anisotropic momentum distribution [11, 12].

- The photino self-energy 45 has a structure identical to the electron self-energy (38) and thus the spectra of collective excitations are also identical. When the plasma momentum distribution is anisotropic and unstable photon modes occur, the photino modes remain stable. Supersymmetry does not change anything here. 
- The selectron self-energy 60 is independent of momentum, it is negative and real. Therefore, $\tilde{\Sigma}$ can be written as $\tilde{\Sigma}=-m_{\text {eff }}^{2}$ where $m_{\text {eff }}$ is the effective selectron mass. Then, the solutions of dispersion equation 10 are $E_{p}= \pm \sqrt{m_{\mathrm{eff}}^{2}+\mathbf{p}^{2}}$.

We conclude our considerations by saying that the collective modes in ultrarelativistic plasma of $\mathcal{N}=1$ SUSY QED are essentially the same as in ultrarelativistic electromagnetic plasma of electrons, positrons and photons.

\section{Acknowledgments}

We are very grateful to Margaret Carrington for helpful correspondence. This work was partially supported by the Polish Ministry of Science and Higher Education under grants N N202 204638 and 667/N-CERN/2010/0.

\section{Appendix: Green's functions of Keldysh-Schwinger formalism}

We present here the retarded, advanced and unordered Green's functions which are usually labeled with the indices ,,$+->,<$, respectively. The form of these functions for free non-equilibrium fields can be found in e.g. 25].

\section{Photons}

The functions of interest for the free electromagnetic field in the Feynman gauge are

$$
\begin{aligned}
D_{\mu \nu}^{ \pm}(p) & =-\frac{g_{\mu \nu}}{p^{2} \pm i \operatorname{sgn}\left(p_{0}\right) 0^{+}}, \\
D_{\mu \nu}^{>}(p) & =\frac{i \pi g_{\mu \nu}}{E_{p}}\left(\delta\left(E_{p}-p_{0}\right)\left[f_{\gamma}(\mathbf{p})+1\right]+\delta\left(E_{p}+p_{0}\right) f_{\gamma}(-\mathbf{p})\right), \\
D_{\mu \nu}^{<}(p) & =\frac{i \pi g_{\mu \nu}}{E_{p}}\left(\delta\left(E_{p}-p_{0}\right) f_{\gamma}(\mathbf{p})+\delta\left(E_{p}+p_{0}\right)\left[f_{\gamma}(-\mathbf{p})+1\right]\right), \\
D_{\mu \nu}^{\mathrm{sym}}(p) & \equiv D_{\mu \nu}^{>}(p)+D_{\mu \nu}^{<}(p)=\frac{i \pi g_{\mu \nu}}{E_{p}}\left(\delta\left(E_{p}-p_{0}\right)\left[2 f_{\gamma}(\mathbf{p})+1\right]+\delta\left(E_{p}+p_{0}\right)\left[2 f_{\gamma}(-\mathbf{p})+1\right]\right),
\end{aligned}
$$

where $f_{\gamma}(\mathbf{p})$ is the distribution function of photons which are assumed to be unpolarized. The function is normalized in such a way that the photon density is given as

$$
n_{\gamma}=2 \int \frac{d^{3} p}{(2 \pi)^{3}} f_{\gamma}(\mathbf{p})
$$

where the factor of 2 takes into account two photon spin states.

One checks that the functions A.1, A.2, A.3 obey the required identity

$$
D_{\mu \nu}^{>}(p)-D_{\mu \nu}^{<}(p)=D_{\mu \nu}^{+}(p)-D_{\mu \nu}^{-}(p) .
$$

The left-hand side of Eq. A.6) equals

$$
D_{\mu \nu}^{>}(p)-D_{\mu \nu}^{<}(p)=\frac{i \pi g_{\mu \nu}}{E_{p}}\left(\delta\left(E_{p}-p_{0}\right)-\delta\left(E_{p}+p_{0}\right)\right)=2 i \pi g_{\mu \nu} \delta\left(p^{2}\right)\left(\Theta\left(p_{0}\right)-\Theta\left(-p_{0}\right)\right) .
$$

Using the well-known relation

$$
\frac{1}{x \pm i 0^{+}}=\mathcal{P} \frac{1}{x} \mp i \pi \delta(x),
$$

one immediately shows that the right-hand side of Eq. A.6 equals the expression A.7. 


\section{Electrons}

The functions for the free massless electron field are

$$
\begin{aligned}
S^{ \pm}(p) & =\frac{\not p}{p^{2} \pm i \operatorname{sgn}\left(p_{0}\right) 0^{+}}, \\
S^{>}(p) & =\frac{i \pi}{E_{p}} \not p\left(\delta\left(E_{p}-p_{0}\right)\left[f_{e}(\mathbf{p})-1\right]+\delta\left(E_{p}+p_{0}\right) \bar{f}_{e}(-\mathbf{p})\right), \\
S^{<}(p) & =\frac{i \pi}{E_{p}} \not p\left(\delta\left(E_{p}-p_{0}\right) f_{e}(\mathbf{p})+\delta\left(E_{p}+p_{0}\right)\left[\bar{f}_{e}(-\mathbf{p})-1\right]\right), \\
S^{\operatorname{sym}}(p) & \equiv S^{>}(p)+S^{<}(p)=\frac{i \pi}{E_{p}} \not p\left(\delta\left(E_{p}-p_{0}\right)\left[2 f_{e}(\mathbf{p})-1\right]+\delta\left(E_{p}+p_{0}\right)\left[2 \bar{f}_{e}(-\mathbf{p})-1\right]\right),
\end{aligned}
$$

where $f_{e}(\mathbf{p})$ and $\bar{f}_{e}(\mathbf{p})$ are the distribution functions of electrons and of positrons, respectively. We assume here that both electrons and positrons are unpolarized. The distribution functions are normalized in such a way that the electron density equals

$$
n_{e}=2 \int \frac{d^{3} p}{(2 \pi)^{3}} f_{e}(\mathbf{p}),
$$

where the factor of 2 takes into account two spin states of each electron. The functions A.9, A.10, A.11) are checked to obey the identity $S^{>}(p)-S^{<}(p)=S^{+}(p)-S^{-}(p)$.

\section{Photinos}

The functions for the free photino field read

$$
\begin{aligned}
\tilde{D}^{ \pm}(p) & =\frac{\not p}{p^{2} \pm i \operatorname{sgn}\left(p_{0}\right) 0^{+}} \\
\tilde{D}^{>}(p) & =\frac{i \pi}{E_{p}} \not p\left(\delta\left(E_{p}-p_{0}\right)\left[f_{\tilde{\gamma}}(\mathbf{p})-1\right]+\delta\left(E_{p}+p_{0}\right) f_{\tilde{\gamma}}(-\mathbf{p})\right), \\
\tilde{D}^{<}(p) & =\frac{i \pi}{E_{p}} \not p\left(\delta\left(E_{p}-p_{0}\right) f_{\tilde{\gamma}}(\mathbf{p})+\delta\left(E_{p}+p_{0}\right)\left[f_{\tilde{\gamma}}(-\mathbf{p})-1\right]\right), \\
\tilde{D}^{\operatorname{sym}}(p) & \equiv \tilde{D}^{>}(p)+\tilde{D}^{<}(p)=\frac{i \pi}{E_{p}} \not p\left(\delta\left(E_{p}-p_{0}\right)\left[2 f_{\tilde{\gamma}}(\mathbf{p})-1\right]+\delta\left(E_{p}+p_{0}\right)\left[2 f_{\tilde{\gamma}}(-\mathbf{p})-1\right]\right),
\end{aligned}
$$

where $f_{\tilde{\gamma}}(\mathbf{p})$ is the distribution function of photinos which are assumed to be unpolarized. The function is normalized in such a way that the photino density is given as

$$
n_{\tilde{\gamma}}=2 \int \frac{d^{3} p}{(2 \pi)^{3}} f_{\tilde{\gamma}}(\mathbf{p}),
$$

where the factor of 2 takes into account two photino spin states. One checks that the required relation $\tilde{D}^{>}(p)-\tilde{D}^{<}(p)=$ $\tilde{D}^{+}(p)-\tilde{D}^{-}(p)$ is satisfied.

\section{Selectrons}

The functions for the free selectron field are

$$
\begin{aligned}
\tilde{S}^{ \pm}(p) & =\frac{1}{p^{2} \pm i \operatorname{sgn}\left(p_{0}\right) 0^{+}}, \\
\tilde{S}^{>}(p) & =-\frac{i \pi}{E_{p}}\left(\delta\left(E_{p}-p_{0}\right)\left[f_{s}(\mathbf{p})+1\right]+\delta\left(E_{p}+p_{0}\right) \bar{f}_{s}(-\mathbf{p})\right), \\
\tilde{S}^{<}(p) & =-\frac{i \pi}{E_{p}}\left(\delta\left(E_{p}-p_{0}\right) f_{s}(\mathbf{p})+\delta\left(E_{p}+p_{0}\right)\left[\bar{f}_{s}(-\mathbf{p})+1\right]\right), \\
\tilde{S}^{\operatorname{sym}}(p) & \equiv \tilde{S}^{>}(p)+\tilde{S}^{<}(p)=-\frac{i \pi}{E_{p}}\left(\delta\left(E_{p}-p_{0}\right)\left[2 f_{s}(\mathbf{p})+1\right]+\delta\left(E_{p}+p_{0}\right)\left[2 \bar{f}_{s}(-\mathbf{p})+1\right]\right),
\end{aligned}
$$


where $f_{s}(\mathbf{p})$ is the distribution function of left or right selectrons and $\bar{f}_{s}(\mathbf{p})$ is the distribution function of left or right antiselectrons. We assume that the distribution functions of left and right (anti-)selectrons are equal to each other. The functions A.19 A.20, A.21 obey the identity $\tilde{S}^{>}(p)-\tilde{S}^{<}(p)=\tilde{S}^{+}(p)-\tilde{S}^{-}(p)$.

[1] O. Aharony, S. S. Gubser, J. M. Maldacena, H. Ooguri and Y. Oz, Phys. Rept. 323, 183 (2000).

[2] I. R. Klebanov, arXiv:hep-th/0009139.

[3] D. T. Son and A. O. Starinets, Ann. Rev. Nucl. Part. Sci. 57, 95 (2007).

[4] R. A. Janik, arXiv:1003.3291 [hep-th].

[5] S. Caron-Huot, P. Kovtun, G. D. Moore, A. Starinets and L. G. Yaffe, JHEP 0612, 015 (2006).

[6] S. Caron-Huot and G. D. Moore, JHEP 0802, 081 (2008).

[7] J. P. Blaizot, E. Iancu, U. Kraemmer and A. Rebhan, JHEP 0706, 035 (2007).

[8] P. M. Chesler and A. Vuorinen, JHEP 0611, 037 (2006).

[9] P. M. Chesler, A. Gynther and A. Vuorinen, JHEP 0909, 003 (2009).

[10] St. Mrówczyński and M. H. Thoma, Ann. Rev. Nucl. Part. Sci. 57, 61 (2007).

[11] St. Mrówczyński, Phys. Rev. D 65, 117501 (2002).

[12] B. Schenke and M. Strickland, Phys. Rev. D 74, 065004 (2006).

[13] M. H. Thoma, in Quark-Gluon Plasma 2, edited by R.C. Hwa (World Scientific, Singapore, 1995).

[14] J. P. Blaizot and E. Iancu, Phys. Rept. 359, 355 (2002).

[15] U. Kraemmer and A. Rebhan, Rept. Prog. Phys. 67, 351 (2004).

[16] St. Mrówczynski and M. H. Thoma, Phys. Rev. D 62, 036011 (2000).

[17] J. C. Taylor and S. M. H. Wong, Nucl. Phys. B 346, 115 (1990).

[18] J. Frenkel and J. C. Taylor, Nucl. Phys. B 374, 156 (1992).

[19] E. Braaten and R. D. Pisarski, Phys. Rev. D 45, 1827 (1992).

[20] J. P. Blaizot and E. Iancu, Nucl. Phys. B 417, 608 (1994).

[21] P. F. Kelly, Q. Liu, C. Lucchesi and C. Manuel, Phys. Rev. D 50, 4209 (1994).

[22] R. D. Pisarski, arXiv:hep-ph/9710370

[23] St. Mrówczyński, A. Rebhan and M. Strickland, Phys. Rev. D 70, 025004 (2004).

[24] T. Binoth, E. W. N. Glover, P. Marquard and J. J. van der Bij, JHEP 0205, 060 (2002).

[25] St. Mrówczyński and U. W. Heinz, Annals Phys. 229, 1 (1994).

[26] U. Kraemmer, A. K. Rebhan and H. Schulz, Annals Phys. 238, 286 (1995).

[27] M. Le Bellac, Thermal Field Theory (Cambridge University Press, Cambridge, 2000). 